\title{
Minimal Residual Disease in Hematological Malignancies
}

\author{
Pia Raanani, Tel Hashomer/Tel Aviv Andreas Hochhaus, Mannheim
}

Do you prefer quality to quantity? This question has been asked for many years. It is well known that mathematics is the science of pure quantity but what about medicine? For many years, counting cells and identifying them by microscopic inspection have determined the number of normal or abnormal cells in hematological and nonhematological malignancies. During the last decade, several studies have shown that detection and quantification of residual tumor cells significantly correlate with clinical outcome in several types of hematological malignancies. In particular the quantitative measurement of the decrease of the leukemic cell load during the first phases of treatment has a high prognostic value [1]. Detection of minimal residual disease (MRD) is now becoming routinely implemented in treatment protocols and is increasingly used for guiding therapy and for evaluation of new treatment modalities [2].

Methods to detect MRD include technologies designed to detect residual malignant cells beyond the sensitivity of conventional approaches like for example morphology and banding cytogenetics in leukemia. A wide variety of techniques have been developed. The choice of the best method for the particular clinical situation certainly depends on the biology of the individual malignancy, i.e. on the determination of specific markers, which are useful to differentiate between leukemic cells and normal hematopoiesis in leukemic patients. These markers include leukocyte differentiation antigens, fusion transcripts, transcripts overexpressed by mutated or nonmutated genes, rearranged genes, and individual markers, like polymorphic repetitive DNA sequences.

In this issue we sought to provide a comprehensive overview on the major technologies for the detection of MRD and their clinical applications. Campana and Coustan-Smith critically discuss advantages and disadvantages of flow cytometry methods in acute leukemias. A special direction of MRD detection is the differentiation between donor and recipient hematopoiesis after allogeneic stem cell transplantation as discussed by Thiede et al. The authors also reviewed the technical issues, advantages and limitations of the methods currently used for chimerism analysis. Adding the morphological analysis of small populations of cells to malignant or recipient-associated markers may improve the accuracy of chimerism and MRD testing, and delineate their clinical significance. Trakhtenbrot et al. introduce the simultaneous analysis of morphology, immunophenotyping and FISH on the same cell, i.e. a multiparametric scanning system. For the comparability of results, an agreement should be reached between various laboratories and different multicenter studies on sample source and volumes obtained (or minimum cell counts) for RT-PCR analyses and regarding the general methods of cell purification, RNA extraction and cDNA synthesis. Rigorous, internationally accepted controls need to be implemented. Müller et al. investigated the impact of preanalytical factors and their standardization. Their review introduces some important considerations for the implementation of RT-PCR-based MRD

\section{KARGER \\ (C) 2004 S. Karger AG, Basel}


endpoints into clinical trials. Specific aspects of childhood acute lymphoblastic leukemia (ALL) are elucidated by Izraeli and Waldman. Fusion transcripts or overexpressed genes, like the Wilms tumor gene WT1, represent a common target for MRD analysis in acute myelogenous leukemia (AML). Current standards and their clinical implications are demonstrated by Raanani and Ben-Bassat as well as the dilemmas and unresolved issues in the interpretation of MRD in AML patients. Recently, the genomic structure of acute promyelocytic leukemia (APL) has been described. Structural factors for the pathogenesis of APL and their implications for MRD monitoring are reviewed by Reiter et al. FLT3 length mutations in AML are considered as prognostic markers as well as markers for MRD studies and are also targets for novel treatment modalities. The current view in this respect is summarized by Schnittger et al. Reduced intensity stem cell transplantation techniques are based on individualized additional immunotherapy depending on exact and rapid MRD monitoring. Shimoni and Nagler outline clinical and methodological aspects of MRD in nonmyeloablative transplantations. MRD monitoring in multiple myeloma is part of purging studies of stem cell aphaeresis and evaluation of novel therapies for this disease. The clinical considerations and molecular data are summarized by Rasmussen et al. The clinical need for molecular endpoints has become even more apparent with the introduction of imatinib in the therapy of chronic myelogenous leukemia (CML) and BCR-ABL-positive ALL. A systematic quantitative PCR monitoring emphasized the prognostic impact of residual BCR-ABL transcripts in CML patients [3, 4]. Paschka et al. discuss the clinical applications and standardized approaches for treatment surveillance of CML patients in the imatinib era. The majority of malignant lymphoproliferative disorders display clonal rearrangement of the antigen receptor genes suitable for detection by PCR. According to Brüggemann et al. MRD-based intensification, modification of treatment and guidance of maintenance treatments should be the goal of current clinical studies to determine the prognostic significance of MRD in chronic lymphocytic leukemia and non-Hodgkin's lymphomas. The WT1 gene is considered a new universal marker for MRD detection and quantification in myeloid malignancies and myelodysplastic syndromes. Cilloni and Saglio review molecular studies using WT1 for the follow-up of these patients.

Currently, PCR-based methods represent the most widely accepted technologies for MRD detection. Over the past 15 years, PCR techniques and implications have been optimized and improved. In view of the limited val- ue of qualitative PCR for treatment surveillance, quantitative PCR assays were developed. Competitive RT-PCR was employed to monitor CML patients after stem cell transplantation $[5,6]$ or treatment with interferon alpha [7], and patients with acute leukemias harboring specific fusion transcripts (CBF $\beta-M Y H 11$ [8]; AML1-ETO [9, 10]). Despite considerable specificity and sensitivity, these methods were cumbersome and time-consuming and were therefore only employed in specialized laboratories.

With the introduction of real-time PCR methodologies and machines quantification of residual disease has been simplified. Results of real-time PCR were readily comparable with competitive PCR data $[11,12]$. Thus, quantification of residual disease has been developed as a major diagnostic tool in most studies focusing on treatment optimization in leukemias characterized by fusion transcripts. A variety of real-time PCR instruments are available and different approaches can be applied. To reach reproducible, sensitive and standardized quantitative PCR data, important prerequisites should be considered: (1) The sensitivity that can be obtained in RT-PCR analyses depends on the number of cells and the total amount of RNA analyzed and on the use of a single or nested PCR approach. (2) Unstabilized anticoagulated blood can be processed even in multicenter trials, when processing is guaranteed within $36 \mathrm{~h}$. As an alternative, bedside RNA stabilization could be made available for multicenter studies with central analysis [13]. (3) The level of target gene transcripts per volume cDNA should be related to the expression of a standard gene.

However, the heterogeneity of the preanalytical environment of PCR machines and methods, and of the methods to calculate the final results causes confusion among patients, treating physicians and laboratories. The universal acceptance of real-time PCR urgently demands standardization of nomenclature and technologies. At present, at least seven different systems are commercially available using three different methods of fluorescence labeling [2]. However, adjustment of the protocols, standardization of preanalytical considerations and of the methods to calculate the final transcript ratio should result in comparable data.

In an attempt to reach an agreement on the minimal requirements for standardization of real-time PCR analyses in leukemias, new international studies have recently been launched. A significant step forward in this direction has been achieved by the recent creation of the European LeukemiaNet. A particular work package within this network has been assigned to MRD analysis in leukemias. 
Standardized PCR protocols will serve as a methodological reference $[14,15]$ and the progress in the development of PCR machines, software and biochemical assays will certainly lead to further improvement.

Methodological aspects and clinical applications of MRD monitoring and standardization strategies are the major topics of this issue - 'from bench to bedside'. We tried to give a comprehensive overview on the significance of MRD in the evaluation, treatment and follow-up of hematological malignancies. We are most grateful to our international colleagues for their major contributions to the subject.

\section{References}

1 van Dongen JJM, MacIntyre EA, Gabert JA, Delabesse E, Rossi V, Saglio G, Gotardi E, Rambaldi A, Dotti G, Griesinger F, Parreira A, Gameiro P, Gonzalez Diaz M, Malec M, Langerak AW, San Miguel JF, Biondi A: Standardized RT-PCR analysis of fusion gene transcripts from chromosome aberrations in acute leukemia for detection of minimal residual disease. Leukemia 1999;13:1901-1928.

2 van der Velden VHJ, Hochhaus A, Cazzaniga G, Szczepanski T, Gabert J, van Dongen JJM: Detection of minimal residual disease in hematologic malignancies by real-time quantitative PCR: Principles, approaches, and laboratory aspects. Leukemia 2003;17:1013-1034.

3 Hughes TP, Kaeda J, Branford S, Rudzki Z, Hochhaus A, Hensley ML, Gathmann I, Bolton AE, van Hoomissen IC, Goldman JM, Radich JP, for the International Randomized Study of Interferon versus STI571 (IRIS) Study Group: Frequency of major molecular responses to imatinib or interferon alfa plus cytarabine in newly diagnosed chronic myeloid leukemia. N Engl J Med 2003;349:1423-1432.

4 Müller MC, Gattermann N, Lahaye T, Deininger MWN, Berndt A, Fruehauf S, Neubauer A, Fischer T, Hossfeld DK, Schneller F, Krause SW, Nerl C, Sayer HG, Ottmann OG, Waller C, Aulitzky W, le Coutre P, Freund M, Merx K, Paschka P, König $H$, Kreil S, Berger U, Gschaidmeier H, Hehlmann R, Hochhaus A: Dynamics of BCR-ABL mRNA expression in first line therapy of chronic myelogenous leukemia patients with imatinib or interferon $\alpha /$ araC. Leukemia 2003;17:2392-2400.

5 Cross NCP, Feng L, Chase A, Bungey J, Hughes TP, Goldman JM: Competitive polymerase chain reaction to estimate the number of BCR-ABL transcripts in chronic myeloid leukemia patients after bone marrow transplantation. Blood 1993;82:1929-1936.
6 Lion T, Henn T, Gaiger A, Kalhs P, Gadner H: Early detection of relapse after bone marrow transplantation in patients with chronic myelogenous leukaemia. Lancet 1993;341:275276.

7 Hochhaus A, Reiter A, Saussele S, Reichert A, Emig M, Kaeda J, Schultheis B, Berger U, Shepherd PCA, Allan NC, Hehlmann R, Goldman JM, Cross NCP, for the German CML Study Group and the UK MRC CML Study Group: Molecular heterogeneity in complete cytogenetic responders after interferon-alpha therapy for chronic myelogenous leukemia: Low levels of minimal residual disease are associated with continuing remission. Blood 2000; 95:62-66.

8 Seale JR, Varma S, Swirsky DM, Pandolfi PP, Goldman JM, Cross NC: Quantification of PML-RAR alpha transcripts in acute promyelocytic leukaemia: Explanation for the lack of sensitivity of RT-PCR for the detection of minimal residual disease and induction of the leukaemia-specific mRNA by alpha interferon. $\mathrm{Br}$ J Haematol 1996;95:95-101.

9 Tobal K, Newton J, Macheta M, Chang J, Morgenstern G, Evans PA, Morgan G, Lucas GS, Liu Yin JA: Molecular quantitation of minimal residual disease in acute myeloid leukemia with $\mathrm{t}(8 ; 21)$ can identify patients in durable remission and predict clinical relapse. Blood 2000;95:815-819.

10 Wattjes MP, Krauter J, Nagel S, Heidenreich O, Ganser A, Heil G: Comparison of nested competitive RT-PCR and real-time RT-PCR for the detection and quantification of AML1/ MTG8 fusion transcripts in $\mathrm{t}(8 ; 21)$ positive acute myelogenous leukemia. Leukemia 2000; 14:329-335.
11 Emig M, Saussele S, Wittor H, Weisser A, Reiter A, Willer A, Berger U, Hehlmann R, Cross NCP, Hochhaus A: Accurate and rapid analysis of residual disease in patients with CML using specific fluorescent hybridization probes for real time quantitative RT-PCR. Leukemia 1999;13:1825-1832.

12 Guo JQ, Lin H, Kantarjian H, Talpaz M, Champlin R, Andreef M, Glassman A, Arlinghaus RB: Comparison of competitive-nested and real-time PCR in detecting BCR-ABL fusion transcripts in chronic myeloid leukemia patients. Leukemia 2002;16:2447-2453.

13 Müller MC, Merx K, Weisser A, Kreil S, Lahaye T, Hehlmann R, Hochhaus A: Improvement of molecular monitoring of residual disease in leukemias by bedside RNA stabilization. Leukemia 2002;16:2395-2399.

14 Gabert J, Beillard E, van der Velden VHJ, Bi W, Grimwade D, Pallisgaard N, Barbany G, Cazzaniga G, Cayuela JM, Cavé H, Pane F, Aerts JLE, de Micheli D, Thirion X, Pradel V, González M, Viehmann S, Malec M, Saglio G, van Dongen JJM: Standardization and quality control studies of 'real-time' quantitative reverse transcriptase polymerase chain reaction (RQ-PCR) of fusion gene transcripts for residual disease detection in leukemia - A Europe Against Cancer Program. Leukemia 2003;17: 2318-2357.

15 Beillard E, Pallisgaard N, van der Velden VHJ, Bi W, Dee R, van der Scoot E, Delabesse E, Macintyre E, Gottardi E, Saglio G, Watzinger F, Lion T, van Dongen JJM, Hokland P, Gabert $\mathrm{J}$ : Evaluation of candidate control genes for diagnosis and residual disease detection in leukemic patients using 'real-time' quantitative reverse-transcriptase polymerase chain reaction (RQ-PCR) - A Europe Against Cancer Program. Leukemia 2003;17:2474-2486. 